\title{
Hypertension and Associated Neurological and Non-Neurological Symptoms in Different Age Groups: A Cross-Sectional Observational Study among Hypertensive Patients
}

\author{
Ayesha $\mathbf{S I}^{1}$, Asra $\mathrm{T}^{2}$, Madiha $\mathrm{A}^{3}$, Aileeya $\mathrm{F}^{4}$, Zarghoona $\mathrm{W}^{5}$, Adnan $\mathrm{A}^{6}$, Neeta $\mathbf{M}^{7^{*}}$, Syed Muhammad $\mathrm{ZHN}^{8}$,
} Sana $\mathrm{N}^{9}$ and Muhammad $\mathrm{A}^{10}$

${ }^{1}$ MBBS, Baqai Medical University, Karachi, Pakistan

${ }^{2}$ MBBS, Medical Officer, Baqai Medical University, Karachi, Pakistan

${ }^{3}$ MBBS, Dow University of Health Sciences, Karachi, Pakistan

${ }^{4}$ MBBS, House Officer, Abbasi Shaheed Hospital, Karachi, Pakistan

${ }^{5}$ MBBS, M. Assistant, Musavvir Stem cell clinic and pathology laboratory

${ }^{6}$ MBBS, Senior Lecturer, Department of Physiology, Altibri Medical College Karachi, Karachi, Pakistan

${ }^{7}$ Manager Medical Affairs and Clinical Research Hilton Pharma Pvt ltd, Pakistan

${ }^{8}$ MBBS, MSBE Assistant Professor, Department of Community Medicine, Baqai Medical University, Karachi, Pakistan

${ }^{9}$ MBBS, MPH, Assistant Professor, Department of Community Medicine, Avicenna Medical College, Lahore, Pakistan

${ }^{10}$ MBBS, Resident Memon Hospital, Karachi, Pakistan

Received: October 09, 2017; Accepted: October 16, 2017; Published: November 02, 2017

*Corresponding author: Neeta Maheshwary, Manager Medical Affairs and Clinical Research, Hilton Pharma Pvt ltd, Pakistan,

E-mail:neeta_maheshwary@yahoo.com

\begin{abstract}
Background: Hypertension is considered to be one of the most common chronic diseases afflicting the world population that is associated with increased mortality. It is estimated that more than a quarter of the world's adult population had hypertension in the year 2000 , and that such prevalence increases with age all over the world.

Objective: To identify and compare the hypertension associated neurological and non-neurological signs and symptoms among different age groups.

Methods: A cross-sectional multicenter study was carried out among patients with self-reported history of hypertension and on antihypertensive medication. The written informed consent was obtained from the patients after taking ethical approval. A total of 174 patients aged 18 years or above were included in the study using convenient sampling technique and the duration of study was from Jan 2017 till June 2017. Patients were divided in two groups according to their ages i.e. $\leq 40$ years and $>40$ years old. A detailed history was taken from each patient about hypertension associated symptoms with the help of a structured questionnaire. Blood pressure was measured using sphygmomanometer with stethoscope to assess hypertension level. The patients with history of diabetes, thyrotoxicosis and liver diseases were excluded from the study. Date was analyzed by using SPSS version 20. Chi square and fisher exact test was used to assess the significance.
\end{abstract}

Results: A higher percentage of the patients in the $>40$ years age group had severe systolic and diastolic hypertension than patients in the $\leq 40$ years age group $(4.0 \%$ vs. $1.4 \%$ and $9.0 \%$ vs. $1.4 \%$ respectively).Among the patients aged $\leq 40$ years only fatigue ( $p=0.049)$ was significantly associated with systolic blood pressure while among patients aged $>40$ years severity of headache $(p=0.026)$, dyspnea $(p=0.022)$, fatigue ( $p=0.003)$ and confusion $(\mathrm{p}=0.006)$ were significantly associated with systolic blood pressure whereas edema $(\mathrm{p}=0.016)$ and fatigue $(\mathrm{p}=0.019)$ were significantly associated with diastolic blood pressure.

Conclusion:The symptoms significantly associated with systolic or diastolic hypertension increased with the increasing age of the patients. These findings give us local evidence emphasizing the need to focus more on the management of older hypertensive patients.

Keywords: Neurological and Non-neurological symptoms; Hypertension; Age Groups 


\section{Introduction}

Hypertension is considered to be one of the most common chronic diseases afflicting the world population that is associated with increased mortality.[1] The comparative Risk Assessment Collaborating Group has identified hypertension as the leading global risk factor for mortality and as the third leading risk factor for disease burden.[2] One of the most common causes of death due to Hypertension is the cardiovascular disease making hypertension the most common reversible risk factor for cardiovascular diseases.[3,4] It is estimated that more than a quarter of the world's adult population- 0.972 billionhad hypertension in the year 2000, that both men and women have similar overall prevalence of hypertension, and that such prevalence increases with age all over the world. Global hypertension prevalence is currently $26 \%$ which is expected to rise to $29 \%$ by the year 2025 . The predictions for 2025 are based on the assumption that the country, age, and sex specific prevalence estimates will remain constant.[5] According to the World Health Organization, the total prevalence of high blood pressure in Pakistan is estimated to be $25.2 \%$ (25.6\% in males and $24.8 \%$ in females).[6]

Hypertension is defined as a systolic blood pressure (SBP) of $140 \mathrm{mmHg}$ or more, or a diastolic blood pressure (DBP) of $90 \mathrm{mmHg}$ or more, or taking antihypertensive medication.[7] Based on the recommendations of the Seventh Report of the Joint national committee on Prevention, Detection, Evaluation, and Treatment of High Blood Pressure (JNC 7), Blood Pressure for adults aged 18 years or older has been classified into four categories as normal, pre-hypertension, stage 1 and stage 2 . Normal indicates a systolic blood pressure of $<120 \mathrm{mmHg}$; and a diastolic $<80 \mathrm{mmHg}$. Pre-hypertension means a systolic blood pressure of 120-139 mmHg; while a diastolic of 80-89 $\mathrm{mmHg}$. Stage 1 reads a systolic of 140-159 mmHg; and a diastolic of 90$99 \mathrm{mmHg}$. Stage 2 is considered to be severe and gives a systolic of $160 \mathrm{mmHg}$ or greater; and a diastolic of $100 \mathrm{mmHg}$ or greater. [8] WHO recognizes three levels of hypertension as levels 1, 2 and 3. Level 1 means a systolic BP between 140 and $159 \mathrm{mmHg}$ and a diastolic BP between 90 and 99, level 2 means a systolic BP between 160 and $179 \mathrm{mmHg}$ and a diastolic BP between 100-109 mmHg while level 3 means a systolic BP of 180 and above and a diastolic BP of $110 \mathrm{mmHg}$ and above.[9]

There are two types of hypertension namely essential and secondary. Essential hypertension can be defined as a rise in blood pressure of unknown cause that increases risk for cerebral, cardiac, and renal events.[10] Secondary hypertension is defined as increased systemic blood pressure due to an identifiable cause. Only $5-10 \%$ of patients suffering from arterial hypertension have a secondary form, whereas the vast majority has essential (idiopathic or primary) hypertension.[11]

Hypertension can present with severe clinical presentation to an even asymptomatic picture depending upon the several factors such as age, gender, and severity of hypertension. Our objective was to find associations between the degree of hypertension and the severity of the associated symptoms among different age groups.

\section{Patients and Methods}

A cross-sectional multicenter study was carried out among patients with self-reported history of hypertension and on antihypertensive medication. After taking ethical approval from Urban Hospital, North Karachi. A total of 174patients aged 18 years or above were included in the study using convenient sampling technique. Patients were divided in two groups according to their ages i.e. $\leq 40$ years and $>40$ years old. A detailed history was taken from each patient about hypertension associated symptoms with the help of a structured questionnaire. The questionnaire was designed based on 12 major or most frequently encountered symptoms by the hypertensive patients. The site, duration, and severity of each symptom were documented. Each symptom was graded from mild to severe to assess the severity of the symptoms. The presence of edema was noted with its laterality and grading. The appearance of chest pain was noted with its severity. The problems of vision were noted and classified according to the loss in field of vision. The history of epistaxis and urinary symptoms were noted with frequency. Few additional symptoms such as nausea, sleep apnea, palpitations, fatigue and confusion were also assessed subjectively. Blood pressure was measured using sphygmomanometer with stethoscope to assess hypertension level.

To classify hypertension, the British Hypertension Society Classification, consistent with the European Hypertension Society and World Health Organization International Society of Hypertension, was used.[12]Similar to WHO classification, it also recognizes three grades of hypertension as grade 1, 2 and 3. Grade I (Mild hypertension) means a systolic BP between 140 and $159 \mathrm{mmHg}$ and a diastolic BP between 90 and 99, Grade II (Moderate hypertension) means a systolic BP between 160 and $179 \mathrm{mmHg}$ and a diastolic BP between 100-109 mmHg whereas Grade III (Severe Hypertension) means a systolic BP of $\geq 180$ $\mathrm{mmHg}$ and a diastolic $\mathrm{BP} \geq 110 \mathrm{mmHg}$.

Patients with history of diabetes, cardiac events, neurological disorders, cluster headache, gastrointestinal disease, visual problems, epistaxis before they were diagnosed with HTN and morbid obesity were excluded from the study. Patients with isolated systolic hypertension were also excluded from the study

For statistical analysis, statistical package for social sciences (SPSS version 21) was used. Chi-square test was performed to assess associations between various study variables and hypertension across different age groups. P-values less than 0.05 were considered statistically significant. The duration of the study was 6 months.

\section{Results}

The study results showed that the mean age of patients aged $\leq 40$ years was $31.19 \pm 7.44$ years whereas that of patients aged $>40$ years was $54.34 \pm 9.13$ years. A majority of patients aged $\leq 40$ years were females whereas that of patients aged $>40$ years were males ( $58.1 \%$ and $62.0 \%$ respectively). A higher percentage of the patients in the $>40$ years age group had severe systolic and diastolic hypertension than patients in the $\leq 40$ years age 
group ( $4.0 \%$ vs. $1.4 \%$ and $9.0 \%$ vs. $1.4 \%$ respectively). Both the mean systolic and diastolic blood pressures were slightly higher in patients aged $\leq 40$ years than those $>40$ years old (148.23 $\pm 13.62 \mathrm{mmHg}$ vs. $146.11 \pm 16.74 \mathrm{mmHg}$ and $92.86 \pm 5.02$ mmHg vs. $91.41 \pm 10.24 \mathrm{mmHg}$ respectively). The mean duration of hypertension was higher in patients belonging to the older age group ( $4.62 \pm 3.51$ years vs. $4.11 \pm 2.99$ years) whereas a slightly higher percentage of them were smokers as well $(15.0 \%$ vs. $8.1 \%$ ). A higher percentage of the older patients had positive clinical history of headache (58.0\% vs. $40.5 \%)$ whereas the most common site of headache was occipital in the younger and temporal in the older age group ( $46.7 \%$ and $24.1 \%$ respectively). The severity of headache was moderate in a majority of the patients belonging to the younger as well as the older age group (56.7\% and $44.8 \%$ respectively). A higher percentage of the patients belonging to the older age group had vertigo than the younger age group ( $44.0 \%$ vs. $21.6 \%$ respectively) whereas a similar majority of both age groups reported its severity to be mild (68.8\% and $68.9 \%$ respectively). A higher percentage of the older patients had edema (28.0\% vs. $17.6 \%)$ but it was seen to be bilateral in a similar percentage of both the younger and the older age groups (61.5\% and $64.3 \%$ respectively). The edema was of moderate grading in a higher percentage of the younger patients (61.5\% vs. $39.3 \%$ ). A higher percentage of the older patients gave a positive history of chest pain (30.0\% vs. $17.6 \%)$ and a higher percentage of those patients in the older age group needed pain relieving medication for it to subside (58.6\% vs. $30.8 \%$ ). A markedly higher percentage of patients belonging to the older age group gave a positive history of vision problems $(54.0 \%$ vs. $24.3 \%$ ) whereas the most common type of vision problem in both the younger and the older patients was dark spots in the field of vision ( $44.4 \%$ and $40.0 \%$ respectively). A markedly higher percentage of patients belonging to the older age group gave a positive history of dyspnea (51.0\% vs. $25.7 \%$ ) whereas most of both the younger and the older patients reported it to be of moderate severity ( $47.4 \%$ and $49 \%$ respectively).Though an almost similar percentage of patients belonging to both the younger and the older age groups suffered from nausea $(21.9 \%$ and $19.0 \%$ respectively),a markedly higher percentage of older patients reported to suffer from sleep apnea, irregular heartbeat/ palpitation, fatigue and confusion(52.0\% and $27.0 \%, 43.0 \%$ and $23.0 \%, 72.0 \%$ and $45.2 \%$ and $62.0 \%$ and $28.8 \%$ respectively) [Table 1].

Table 1: Description of participants' characteristics: Age wise comparison

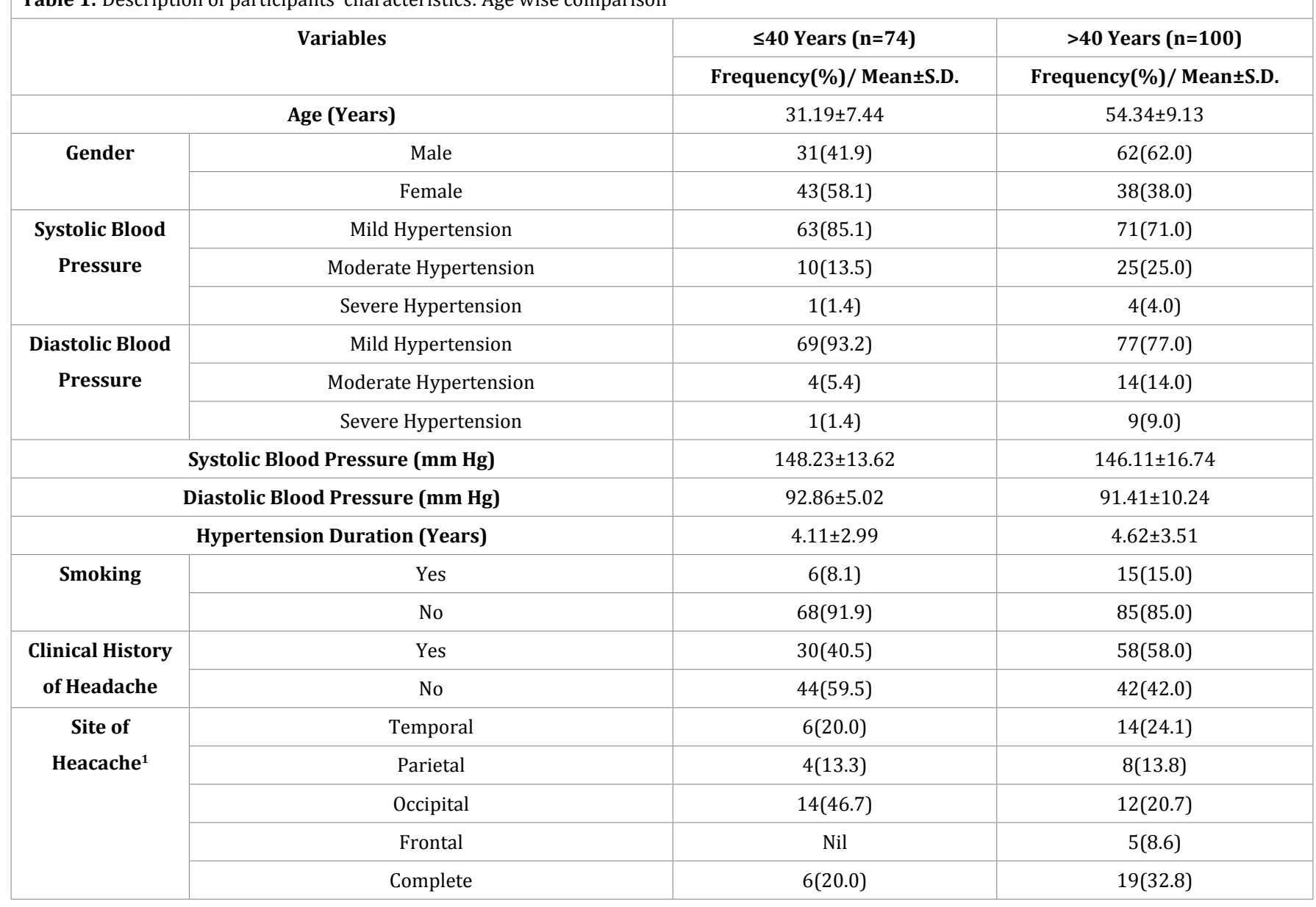


Hypertension and Associated Neurological and Non-Neurological Symptoms in Different Age Groups: A Cross-Sectional Observational Study among Hypertensive Patients

\begin{tabular}{|c|c|c|c|}
\hline \multirow{3}{*}{$\begin{array}{l}\text { Severity of } \\
\text { Headache }^{1}\end{array}$} & Mild & $5(16.6)$ & $9(15.5)$ \\
\hline & Moderate & $17(56.7)$ & $26(44.8)$ \\
\hline & Severe & $8(26.7)$ & $23(39.7)$ \\
\hline \multirow[t]{2}{*}{ Vertigo } & Yes & $16(21.6)$ & $44(44.0)$ \\
\hline & No & $58(78.4)$ & $56(56.0)$ \\
\hline \multirow{3}{*}{$\begin{array}{l}\text { Severity of } \\
\text { Vertigo }^{2}\end{array}$} & Mild & $11(68.8)$ & $31(68.9)$ \\
\hline & Moderate & $5(31.2)$ & $11(24.4)$ \\
\hline & Severe & Nil & $3(6.7)$ \\
\hline \multirow[t]{2}{*}{ Edema } & Yes & $13(17.6)$ & $28(28.0)$ \\
\hline & No & $61(82.4)$ & $72(72.0)$ \\
\hline \multirow{2}{*}{$\begin{array}{l}\text { Laterality of } \\
\text { Edema }^{3}\end{array}$} & Bilateral & $8(61.5)$ & $18(64.3)$ \\
\hline & Unilateral & $5(38.5)$ & $10(35.7)$ \\
\hline \multirow{3}{*}{$\begin{array}{c}\text { Grading of } \\
\text { Bilateral Edema }^{3}\end{array}$} & Mild & $1(7.7)$ & $8(28.6)$ \\
\hline & Moderate & $8(61.5)$ & 11(39.3) \\
\hline & Severe & $4(30.8)$ & $9(32.1)$ \\
\hline \multirow[t]{2}{*}{ Chest Pain } & Yes & $13(17.6)$ & $30(30.0)$ \\
\hline & No & $61(82.4)$ & $70(70.0)$ \\
\hline \multirow{3}{*}{$\begin{array}{c}\text { Severity of Chest } \\
\text { Pain }^{4}\end{array}$} & Improves with rest & $7(53.8)$ & $8(27.6)$ \\
\hline & Needs pain relieving medication & $4(30.8)$ & $17(58.6)$ \\
\hline & Requires hospital visit & $2(15.4)$ & $4(13.8)$ \\
\hline \multirow[t]{2}{*}{ Vision Problems } & Yes & $18(24.3)$ & $54(54.0)$ \\
\hline & No & $56(75.7)$ & $46(46.0)$ \\
\hline \multirow{4}{*}{$\begin{array}{c}\text { Type of Vision } \\
\text { Problem }^{5}\end{array}$} & Loss of Central Vision & $2(11.1)$ & $6(12.0)$ \\
\hline & Loss of Peripheral Vision & $5(27.8)$ & $13(26.0)$ \\
\hline & Dark Spots in field of Vision & $8(44.4)$ & $20(40.0)$ \\
\hline & Pain in Eyes & $3(16.7)$ & $11(22.0)$ \\
\hline \multirow[t]{2}{*}{ Dyspnea } & Yes & $19(25.7)$ & $51(51.0)$ \\
\hline & No & $55(74.3)$ & $49(49.0)$ \\
\hline \multirow{3}{*}{$\begin{array}{l}\text { Dyspnea } \\
\text { Severity }^{6}\end{array}$} & Mild & $4(21.1)$ & $8(15.7)$ \\
\hline & Moderate & $9(47.4)$ & $25(49.0)$ \\
\hline & Severe & $6(31.6)$ & $18(35.3)$ \\
\hline \multirow[t]{2}{*}{ Nausea $^{7}$} & Yes & $16(21.9)$ & $19(19.0)$ \\
\hline & No & $57(78.1)$ & $81(81.0)$ \\
\hline \multirow[t]{2}{*}{ Sleep Apnea } & Yes & $20(27.0)$ & $52(52.0)$ \\
\hline & No & $54(73.0)$ & $48(48.0)$ \\
\hline \multirow{2}{*}{$\begin{array}{c}\text { Irregular } \\
\text { Heartbeat/ } \\
\text { Palpitation }\end{array}$} & Yes & $17(23.0)$ & $43(43.0)$ \\
\hline & No & $57(77.0)$ & $57(57.0)$ \\
\hline \multirow[t]{2}{*}{ Fatigue $^{7}$} & Yes & $33(45.2)$ & $72(72.0)$ \\
\hline & No & $40(54.8)$ & $28(28.0)$ \\
\hline \multirow[t]{2}{*}{ Confusion $^{7}$} & Yes & $21(28.8)$ & $62(62.0)$ \\
\hline & No & $52(71.2)$ & $38(38.0)$ \\
\hline
\end{tabular}


${ }^{1} \mathrm{n}=30$ and 58 in $\leq 40$ years and $>40$ years old respectively
${ }^{2} \mathrm{n}=16$ and 45 in $\leq 40$ years and $>40$ years old respectively
${ }^{3} \mathrm{n}=13$ and 28 in $\leq 40$ years and $>40$ years old respectively
${ }^{4} n=13$ and 29 in $\leq 40$ years and $>40$ years old respectively
${ }^{5} n=18$ and 50 in $\leq 40$ years and $>40$ years old respectively
${ }^{6} n=19$ and 51 in $\leq 40$ years and $>40$ years old respectively
${ }^{7} n=73$ and 100 in $\leq 40$ years and $>40$ years old respectively

The study findings further showed that among the patients aged $\leq 40$ years only fatigue $(\mathrm{p}=0.049)$ was significantly associated with systolic blood pressure where those who had mild systolic hypertension very more likely to suffer from fatigue than those who had moderate/severe systolic hypertension $(50.0 \%$ vs. $18.2 \%$ respectively). None of the other variables were found to be significantly associated with either systolic or diastolic blood pressure in this age group [Table 2].

Among patients aged $>40$ years severity of headache $(p=0.026)$, dyspnea $(p=0.022)$, fatigue $(p=0.003)$ and confusion $(p=0.006)$ were significantly associated with systolic blood pressure where those who had mild systolic hypertension were less likely to have mild/moderate headache, dyspnea, fatigue and confusion than those who had moderate/severe systolic hypertension $(50.0 \%$ vs. $80.0 \%, 43.7 \%$ vs. $69.0 \%, 63.4 \%$ vs. $93.1 \%$ and $53.5 \%$ vs. $82.8 \%$ respectively) whereas edema $(\mathrm{p}=0.016)$ and fatigue $(\mathrm{p}=0.019)$ were significantly associated with diastolic blood pressure where those who had mild diastolic hypertension were less likely to have edema and fatigue than those who had moderate/severe diastolic hypertension $(22.1 \%$ vs. $47.8 \%$ and $66.2 \%$ vs. $91.3 \%$ respectively).None of the other variables were found to be significantly associated with either systolic or diastolic blood pressure in this age group [Table 3].

Table 2: Relationship between blood pressure and patients' signs and symptoms in $\leq 40$ year's age group

\begin{tabular}{|c|c|c|c|c|c|}
\hline \multirow{4}{*}{ Variables } & & \multicolumn{4}{|c|}{$\leq 40$ Years $(n=74)$} \\
\hline & & \multicolumn{2}{|r|}{ SBP } & \multicolumn{2}{|l|}{ DBP } \\
\hline & & Mild Hypertension & $\begin{array}{c}\text { Moderate/Severe } \\
\text { Hypertension }\end{array}$ & Mild Hypertension & $\begin{array}{c}\text { Moderate/Severe } \\
\text { Hypertension }\end{array}$ \\
\hline & & Frequency (\%) & Frequency (\%) & Frequency (\%) & Frequency (\%) \\
\hline \multirow{2}{*}{ Gender } & Male & $26(41.3)$ & $5(45.5)$ & $29(42.0)$ & $2(40.0)$ \\
\hline & Female & $37(58.7)$ & $6(54.5)$ & $40(58.0)$ & $3(60.0)$ \\
\hline \multicolumn{2}{|c|}{$\mathbf{P}$} & \multicolumn{2}{|c|}{$0.523^{*}$} & \multicolumn{2}{|c|}{$0.653^{*}$} \\
\hline \multirow{2}{*}{ Smoking } & Yes & $6(9.5)$ & Nil & $6(8.7)$ & Nil \\
\hline & No & $57(90.5)$ & $11(100)$ & $63(91.3)$ & $5(100)$ \\
\hline \multicolumn{2}{|c|}{$\mathbf{P}$} & \multicolumn{2}{|c|}{$0.367^{*}$} & \multicolumn{2}{|c|}{$0.647^{*}$} \\
\hline $\begin{array}{c}\text { Clinical History of } \\
\text { Headache }\end{array}$ & No & $36(57.1)$ & $8(72.7)$ & $41(59.4)$ & $3(60)$ \\
\hline \multicolumn{2}{|c|}{$\mathbf{P}$} & \multicolumn{2}{|c|}{$0.266^{*}$} & \multicolumn{2}{|c|}{$0.678^{*}$} \\
\hline \multirow{2}{*}{$\begin{array}{l}\text { Severity of } \\
\text { Headache }\end{array}$} & Mild/Moderate & $20(74.1)$ & $2(66.7)$ & $20(71.4)$ & $2(100)$ \\
\hline & Severe & $7(25.9)$ & $1(33.3)$ & $8(28.6)$ & Nil \\
\hline \multicolumn{2}{|c|}{$\mathbf{P}$} & \multicolumn{2}{|c|}{$0.621^{*}$} & \multicolumn{2}{|c|}{$0.531^{*}$} \\
\hline \multirow{2}{*}{ Vertigo } & Yes & $13(20.6)$ & $3(27.3)$ & $15(21.7)$ & $1(20.0)$ \\
\hline & No & $50(79.4)$ & $8(72.7)$ & $54(78.3)$ & $4(80.0)$ \\
\hline \multicolumn{2}{|c|}{$\mathbf{P}$} & \multicolumn{2}{|c|}{$0.44^{*}$} & \multicolumn{2}{|c|}{$0.706^{*}$} \\
\hline \multirow{2}{*}{ Edema } & Yes & $12(19.0)$ & $1(9.1)$ & $13(18.8)$ & Nil \\
\hline & No & $51(81.0)$ & $10(90.9)$ & $56(81.2)$ & $5(100)$ \\
\hline Laterality of Edema & Unilateral & $5(41.7)$ & Nil & $5(38.5)$ & Nil \\
\hline
\end{tabular}

Citation: Ayesha SI, Asra T, et al. (2017) Hypertension and Associated Neurological and Non-Neurological Symptoms in Different 
Hypertension and Associated Neurological and Non-Neurological Symptoms in Different Age Groups: A Cross-Sectional Observational Study among Hypertensive Patients

\begin{tabular}{|c|c|c|c|c|c|}
\hline \multicolumn{2}{|c|}{$\mathbf{P}$} & \multicolumn{2}{|c|}{$0.615^{*}$} & \multicolumn{2}{|c|}{$\ldots}$. \\
\hline \multirow{2}{*}{$\begin{array}{c}\text { Grading of Bilateral } \\
\text { Edema }\end{array}$} & Mild/Moderate & $8(66.7)$ & $1(100)$ & $9(69.2)$ & Nil \\
\hline & Severe & $4(33.3)$ & Nil & $4(30.8)$ & Nil \\
\hline \multicolumn{2}{|c|}{$\mathbf{P}$} & \multicolumn{2}{|c|}{$0.692^{*}$} & \multicolumn{2}{|c|}{$\ldots}$. \\
\hline \multirow{2}{*}{ Chest Pain } & Yes & $12(19.0)$ & $1(9.1)$ & $13(18.8)$ & Nil \\
\hline & No & $51(81.0)$ & $10(90.0)$ & $56(81.2)$ & $5(100)$ \\
\hline \multicolumn{2}{|c|}{$\mathbf{P}$} & \multicolumn{2}{|c|}{$0.38^{*}$} & \multicolumn{2}{|c|}{$0.369 *$} \\
\hline \multirow{2}{*}{ Vision Problems } & Yes & $17(27.0)$ & $1(9.1)$ & $18(26.1)$ & Nil \\
\hline & No & $46(73.0)$ & $10(90.9)$ & $51(73.9)$ & $5(100)$ \\
\hline \multicolumn{2}{|c|}{$\mathbf{P}$} & \multicolumn{2}{|c|}{$0.189^{*}$} & \multicolumn{2}{|c|}{$0.237^{*}$} \\
\hline \multirow{2}{*}{ Dyspnea } & Yes & $17(27.0)$ & $2(18.2)$ & $19(27.5)$ & Nil \\
\hline & No & $46(73.0)$ & $9(81.8)$ & $50(72.5)$ & $5(100)$ \\
\hline \multicolumn{2}{|c|}{$\mathbf{P}$} & \multicolumn{2}{|c|}{$0.422^{*}$} & \multicolumn{2}{|c|}{$0.216^{*}$} \\
\hline \multirow{2}{*}{ Severity of Dyspnea } & Mild/Moderate & $12(70.6)$ & $1(50.0)$ & $13(68.4)$ & Nil \\
\hline & Severe & $5(29.4)$ & $1(50.0)$ & $6(31.6)$ & Nil \\
\hline \multicolumn{2}{|c|}{$\mathbf{P}$} & \multicolumn{2}{|c|}{$0.544^{*}$} & \multicolumn{2}{|c|}{$\ldots$} \\
\hline \multirow{2}{*}{ Nausea } & Yes & $14(22.6)$ & $2(18.2)$ & $15(22.1)$ & $1(20.0)$ \\
\hline & No & $48(77.4)$ & $9(81.2)$ & $53(77.9)$ & $4(80.0)$ \\
\hline \multicolumn{2}{|c|}{$\mathbf{P}$} & \multicolumn{2}{|c|}{$0.549^{*}$} & \multicolumn{2}{|c|}{$0.700^{*}$} \\
\hline \multirow{2}{*}{ Sleep Apnea } & Yes & $17(27.0)$ & $3(27.3)$ & $19(27.5)$ & $1(20.0)$ \\
\hline & No & $46(73.0)$ & $8(72.7)$ & $50(72.5)$ & $4(80.0)$ \\
\hline \multicolumn{2}{|c|}{$\mathbf{P}$} & \multicolumn{2}{|c|}{$0.621^{*}$} & & \\
\hline Irregular & Yes & $15(23.8)$ & $2(18.2)$ & $17(24.6)$ & Nil \\
\hline $\begin{array}{l}\text { Heartbeat/ } \\
\text { Palpitation }\end{array}$ & No & $48(76.2)$ & $9(81.8)$ & $52(75.4)$ & $5(100)$ \\
\hline & & & & & \\
\hline Fotiant & Yes & $31(50.0)$ & $2(18.2)$ & $31(45.6)$ & $2(40.0)$ \\
\hline & No & $31(50.0)$ & $9(81.8)$ & $37(54.4)$ & $3(60.0)$ \\
\hline & & & & & \\
\hline Confucion & Yes & $18(29.0)$ & $3(27.3)$ & $19(27.9)$ & $2(40.0)$ \\
\hline Comtusioni & No & $44(71.0)$ & $8(72.7)$ & $49(72.1)$ & $3(60.0)$ \\
\hline & & & & & \\
\hline *Fisher's Exact Test & & & & & \\
\hline
\end{tabular}

Table 3: Relationship between blood pressure and patients' signs and symptoms in $>40$ year's age group

\begin{tabular}{|c|c|c|c|c|c|}
\hline \multirow{3}{*}{ Variables } & \multicolumn{5}{|c|}{$>40$ Years $(n=100)$} \\
\hline & \multicolumn{2}{|c|}{ SBP } & \multicolumn{2}{|c|}{ DBP } & \multirow[b]{2}{*}{$\begin{array}{c}\text { Moderate/Severe } \\
\text { Hypertension }\end{array}$} \\
\hline & & $\begin{array}{c}\text { Mild } \\
\text { Hypertension }\end{array}$ & $\begin{array}{c}\text { Moderate/Severe } \\
\text { Hypertension }\end{array}$ & $\begin{array}{c}\text { Mild } \\
\text { Hypertension }\end{array}$ & \\
\hline & & Frequency $(\%)$ & Frequency $(\%)$ & Frequency $(\%)$ & Frequency(\%) \\
\hline \multirow{2}{*}{ Gender } & Male & $46(64.8)$ & $16(55.2)$ & $47(61.0)$ & $15(65.2)$ \\
\hline & Female & $25(35.5)$ & $13(44.8)$ & $30(39.0)$ & $8(34.8)$ \\
\hline \multicolumn{2}{|c|}{$\mathbf{P}$} & \multicolumn{2}{|c|}{0.369} & \multicolumn{2}{|c|}{0.717} \\
\hline
\end{tabular}


Hypertension and Associated Neurological and Non-Neurological Symptoms in Different Age Groups: A Cross-Sectional Observational Study among Hypertensive Patients

\begin{tabular}{|c|c|c|c|c|c|}
\hline \multirow{2}{*}{ Smoking } & Yes & $9(12.7)$ & $6(20.7)$ & $10(13.0)$ & $5(21.7)$ \\
\hline & No & $62(87.3)$ & $23(79.3)$ & $67(87.0)$ & 18(78.3) \\
\hline \multicolumn{2}{|l|}{$\mathbf{P}$} & \multicolumn{2}{|c|}{$0.235^{*}$} & \multicolumn{2}{|c|}{$0.236^{*}$} \\
\hline \multirow{2}{*}{$\begin{array}{l}\text { Clinical History of } \\
\text { Headache }\end{array}$} & Yes & $38(53.5)$ & $20(69.0)$ & $43(55.8)$ & $15(65.2)$ \\
\hline & No & $33(46.5)$ & $9(31.0)$ & $34(44.2)$ & $8(34.8)$ \\
\hline \multicolumn{2}{|l|}{$\mathbf{P}$} & \multicolumn{2}{|c|}{0.156} & \multicolumn{2}{|c|}{0.424} \\
\hline \multirow{2}{*}{ Severity of Headache } & Mild/Moderate & $19(50.0)$ & $16(80.0)$ & $23(53.5)$ & $12(80.0)$ \\
\hline & Severe & $19(50.0)$ & $4(20.0)$ & $20(46.5)$ & $3(20.0)$ \\
\hline \multicolumn{2}{|l|}{$\mathbf{P}$} & \multicolumn{2}{|c|}{0.026} & \multicolumn{2}{|c|}{0.071} \\
\hline \multirow{2}{*}{ Vertigo } & Yes & $29(40.8)$ & $15(51.7)$ & $34(44.2)$ & $10(43.5)$ \\
\hline & No & $42(59.2)$ & $14(48.3)$ & $43(55.8)$ & $13(56.5)$ \\
\hline \multicolumn{2}{|l|}{$\mathbf{P}$} & \multicolumn{2}{|c|}{0.32} & \multicolumn{2}{|c|}{0.954} \\
\hline \multirow{2}{*}{ Edema } & Yes & $17(23.9)$ & $11(37.9)$ & $17(22.1)$ & $11(47.8)$ \\
\hline & No & $54(76.1)$ & $18(62.1)$ & $60(77.9)$ & $12(52.2)$ \\
\hline \multicolumn{2}{|l|}{$\mathbf{P}$} & \multicolumn{2}{|c|}{0.157} & \multicolumn{2}{|c|}{0.016} \\
\hline \multirow{2}{*}{ Laterality of Edema } & Bilateral & $11(64.7)$ & $7(63.6)$ & $12(70.6)$ & $6(54.5)$ \\
\hline & Unilateral & $6(35.3)$ & $4(36.4)$ & $5(29.4)$ & $5(45.5)$ \\
\hline \multicolumn{2}{|l|}{$\mathbf{P}$} & \multicolumn{2}{|c|}{$0.632^{*}$} & \multicolumn{2}{|c|}{$0.321^{*}$} \\
\hline \multirow{2}{*}{$\begin{array}{c}\text { Grading of Bilateral } \\
\text { Edema }\end{array}$} & Mild/Moderate & $13(76.5)$ & $6(54.5)$ & $14(77.8)$ & $5(50.0)$ \\
\hline & Severe & $4(23.5)$ & $5(45.5)$ & $4(22.2)$ & $5(50.0)$ \\
\hline $\mathbf{P}$ & & & & & \\
\hline Chongi- & Yes & $19(26.8)$ & $11(37.9)$ & $21(27.3)$ & $9(39.1)$ \\
\hline Cnest Pain & No & $52(73.2)$ & $18(62.1)$ & $56(72.7)$ & $14(60.9)$ \\
\hline $\mathbf{P}$ & & & & & \\
\hline W. & Yes & $35(49.3)$ & $19(65.5)$ & $40(51.9)$ & $14(60.9)$ \\
\hline Vision Prodiems & No & $36(50.7)$ & $10(34.5)$ & $37(48.1)$ & $9(39.1)$ \\
\hline $\mathbf{P}$ & & & & & \\
\hline Dycnno & Yes & $31(43.7)$ & $20(69.0)$ & $37(48.1)$ & $14(60.9)$ \\
\hline Dyspnea & No & $40(56.3)$ & $9(31.0)$ & $40(51.9)$ & $9(39.1)$ \\
\hline $\mathbf{P}$ & & & & & \\
\hline 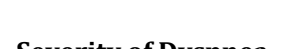 & Mild/Moderate & $19(61.3)$ & $14(70.0)$ & $23(62.2)$ & $10(71.4)$ \\
\hline severity or wyspned & Severe & $12(38.7)$ & $6(30.0)$ & $14(37.8)$ & $4(28.6)$ \\
\hline $\mathbf{P}$ & & & & & \\
\hline Nansog & Yes & $12(16.9)$ & $7(24.1)$ & 13(16.9) & $6(26.1)$ \\
\hline Nausea & No & $59(83.1)$ & $22(75.9)$ & $64(83.1)$ & $17(73.9)$ \\
\hline $\mathbf{P}$ & & & & & \\
\hline S1 & Yes & $33(46.5)$ & $19(65.5)$ & $37(48.1)$ & $15(65.2)$ \\
\hline sieep Apnea & No & $38(53.5)$ & $10(34.5)$ & $40(51.9)$ & $8(34.8)$ \\
\hline $\mathbf{P}$ & & & & & \\
\hline Irregular Heartbeat/ & Yes & $27(38.0)$ & $16(55.2)$ & $31(40.3)$ & $12(52.2)$ \\
\hline Palpitation & No & $44(62.0)$ & $13(44.8)$ & $46(59.7)$ & $11(47.8)$ \\
\hline $\mathbf{P}$ & & & & & \\
\hline
\end{tabular}

Citation: Ayesha SI, Asra T, et al. (2017) Hypertension and Associated Neurological and Non-Neurological Symptoms in Different 

in Different Age Groups: A Cross-Sectional Observational Study among Hypertensive Patients

\begin{tabular}{|c|c|c|c|c|c|}
\hline \multirow{2}{*}{ Fatigue } & Yes & $45(63.4)$ & $27(93.1)$ & $51(66.2)$ & $21(91.3)$ \\
\hline & No & $26(36.6)$ & $2(6.9)$ & $26(33.8)$ & $2(8.7)$ \\
\hline \multicolumn{2}{|c|}{$\mathbf{P}$} & \multicolumn{2}{|c|}{0.003} & \multicolumn{2}{|c|}{0.019} \\
\hline \multirow{2}{*}{ Confusion } & Yes & $38(53.5)$ & $24(82.8)$ & $45(58.4)$ & 17(73.9) \\
\hline & No & $33(46.5)$ & $5(17.2)$ & $32(41.6)$ & $6(26.1)$ \\
\hline \multicolumn{2}{|c|}{$\mathbf{P}$} & \multicolumn{2}{|c|}{0.006} & \multicolumn{2}{|c|}{0.18} \\
\hline
\end{tabular}

\section{Discussion}

The study findings revealed that a higher percentage of the patients in the $>40$ years age group had severe systolic and diastolic hypertension than patients in the $\leq 40$ years age group. Furthermore, and expectedly, the hypertension associated sign and symptom such as smoking, clinical history of headache, vertigo, edema, chest pain, vision problems, dyspnea, sleep apnea, irregular heartbeat/palpitation, fatigue and confusion were found to be more prevalent in the older than in the younger age group.

The study findings further showed that in the younger age group only fatigue was found to be significantly associated with systolic blood pressure and none of the other variables were significantly associated with diastolic blood pressure whereas, as expected, in the older age group a number of signs and symptoms were found to be significantly associated with systolic hypertension, such as severity of headache, dyspnea, fatigue and confusion, as well as with diastolic hypertension, such as edema and fatigue.

As expected, the study results showed that the older patients had higher prevalence of severe systolic and diastolic hypertension than the younger patients, a finding well in line with the published literature. An earlier study also reported the prevalence of hypertension to increase with increasing age with $8 \%$ in patients aged $30-39$ years to $38 \%$ in patients aged $\geq 60$ years.[13] Another study found hypertension prevalence to show an increasing trend with age with $9.5 \%$ in patients aged $15-18$ years to $42 \%$ in patients aged $>60$ years.[14] Another study showed the prevalence of hypertension to increase with increasing age of the study participants.[15] Yet another study reported age to be significantly associated with hypertension. [16]

The study results further showed that the signs and symptoms associated with hypertension increased with the increasing age of the study participants. At younger age, only fatigue was found to have a significant relationship with systolic blood pressure whereas at older age, severity of headache, edema, dyspnea, fatigue and confusion all were found to be significantly associated with either systolic or diastolic hypertension or both. An earlier study reported headache to be significantly more prevalent in hypertensive than in normotensive subjects $(\mathrm{p}<0.05)$ but contrary findings have been reported as well as another study did not report headache to be significantly associated with hypertension (OR 1.02, 95\% CI 0.79 to 1.30 ).[17,18] This difference in findings could be due to different methods of blood pressure measurement in both studies i.e. Direct versus indirect auscultatory method. Similarly, an earlier study also reported dyspnea to be associated with elevated blood pressure though irrespective of age of the study participants.[19] Unlike this study, an earlier study did not report any significant difference in prevalence of weakness among hypertensive and normotensive subjects though this difference in findings could be due to the use of different operational definitions of the terminology involved i.e. Fatigue versus weakness.[17] With regard to the study findings regarding association of edema, fatigue and confusion with hypertension, due to lack of relevant data, a comparison could not be made with the published literature.

\section{Limitation}

The use of convenient sampling method, because of financial and time restraints, was the only limitation of the study.

\section{Conclusion}

The symptoms significantly associated with systolic or diastolic hypertension increased with the increasing age of the patients. These findings give us local evidence emphasizing the need to focus more on the management of older hypertensive patients.

\section{Recommendation}

In the light of the study findings it is recommended that during hypertension screening and management, health care professionals need to specially focus on the patients aged $>40$ years as these patients are more likely to have higher number of hypertension associated sign and symptoms which may require more intensive management and counseling on part of treating physicians.

\section{References}

1. Pandey R, Quan WY, Hong F, Su LJ. Vaccine for Hypertension: Modulating the renin-angiotensin System. International journal of cardiology. 2009; 134(2):160-168; Doi: 10.1016/j.ijcard.2009.03.032

2. Ezzati M, Lopez AD, Rodgers A, Vos T, Danaei G, Aryee M et al. Comparative risk assessment collaborative group: selected major risk factors and global and regional burden of disease. Lancet 2002; 360(9859):1347-1360

3. He J, Whelton PK. Epidemiology and prevention of hypertension. Med Clin North Am 1997; 81(5):1077-1097

4. Whelton PK. Epidemiology of hypertension. Lancet. 1994; 344(8915):101-106 


\section{Hypertensive Patients}

5. Kearney MP, Whelton M, Reynolds K, Muntner P, Whelton KP, Heet J Global burden of hypertension: analysis of worldwide data. Lancet 2005; 365(9455): 217-223

6. Non-communicable Diseases Country Profile 2014. World Health Organization. 2014

7. Roger VL, Go AS, Lloyd-Jones DM, Benjamin EJ, Berry JD, Borden WB et al. Heart disease and stroke statistics--2012 update: a report from the American Heart Association. Circulation. 2012. 125(1):e2-e220; Doi: 10.1161/CIR.0b013e31823ac046

8. Chobanian AV, Bakris GL, Black HR, Cushman WC, Green LA, Izzo JL Jr., et al. The seventh report of the joint national committee on prevention, detection, evaluation, and treatment of high blood pressure: the JNC 7 report. JAMA. 2003; 289(19):2560-2572; Doi: 10.1001/jama.289.19.2560

9. Heidenheim (BW): Paul Hartmann AG; Paul Hartmann AG; c2017. High blood pressure (hypertension). Hartmann international. 2016

10.Messerli FH, Williams B, Ritz E. Essential hypertension. The Lancet. 2007; 370(9587):591-603

11.Mancia G, Fagard R, Narkiewicz K, Redon J, Zanchetti A, Bohm M, et al. 2013 ESH/ ESC Guidelines for the management of arterial hypertension: The Task Force for the management of arterial hypertension of the European Society of Hypertension (ESH) and of the European Society of Cardiology (ESC). European Heart Journal. 2013;34(28): $2159-2219$
12.British Hypertension Society. BHS. 2011

13.Kokiwar PR, Gupta SS, Durge PM. Prevalence of hypertension in a rural community of central India. J Assoc Physicians India. 2012; 60(6):2629

14.Esam MS, Husain AS. Prevalence of Prehypertension and Hypertension in rural Bareilly. Natl J Med Res. 2012; 2(3):291-294

15.Sherif SM, Ahmed ME, Homeida MM. Prevalence of hypertension in an urban community in Sudan. Khartoum Medical Journal. 2012;1(2)

16.Choi KM, Park HS, Han JH, Lee JS, Lee J, Ryu OH, et al. Prevalence of prehypertension and hypertension in a Korean population: Korean National Health and Nutrition Survey 2001. J Hypertens. 2006; 24(8):1515-1521; Doi: 10.1097/01.hjh.0000239286.02389.0f

17.Di Tullio M, Alli C, Avanzini F, Bettelli G, Colombo F, Devoto MA, et al. Prevalence of symptoms generally attributed to hypertension or its treatment: study on blood pressure in elderly outpatients (SPAA). Journal of hypertension. Supplement: official journal of the International Society of Hypertension. J Hypertens Suppl. 1988; 6(1):S87-90

18.Fuchs FD, Gus M, Moreira LB, Moreira WD, Goncalves SC, Nunes $\mathrm{G}$. Headache is not more frequent among patients with moderate to severe hypertension. Journal of human hypertension. 2003 ;17(11):787

19.Karras DJ, Ufberg JW, Harrigan RA, Wald DA, Botros MS, McNamara RM. Lack of relationship between hypertension-associated symptoms and blood pressure in hypertensive ED patients. The American journal of emergency medicine. 2005; 23(2):106-110 